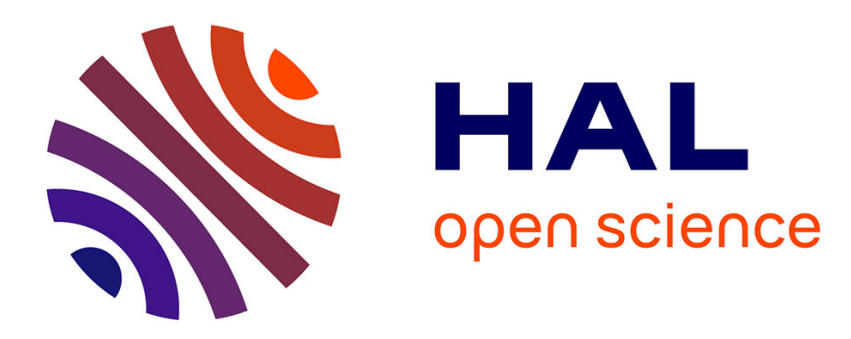

\title{
Theory of stress-strain relations in anisotropic viscoelasticity and relaxation phenomena
}

\author{
Maurice A. Biot
}

\section{To cite this version:}

Maurice A. Biot. Theory of stress-strain relations in anisotropic viscoelasticity and relaxation phenomena. Journal of Applied Physics, 1954, 25 (11), pp.1385-1391. 10.1063/1.1721573 . hal-01368658

\section{HAL Id: hal-01368658 \\ https://hal.science/hal-01368658}

Submitted on 21 Sep 2016

HAL is a multi-disciplinary open access archive for the deposit and dissemination of scientific research documents, whether they are published or not. The documents may come from teaching and research institutions in France or abroad, or from public or private research centers.
L'archive ouverte pluridisciplinaire HAL, est destinée au dépôt et à la diffusion de documents scientifiques de niveau recherche, publiés ou non, émanant des établissements d'enseignement et de recherche français ou étrangers, des laboratoires publics ou privés. 


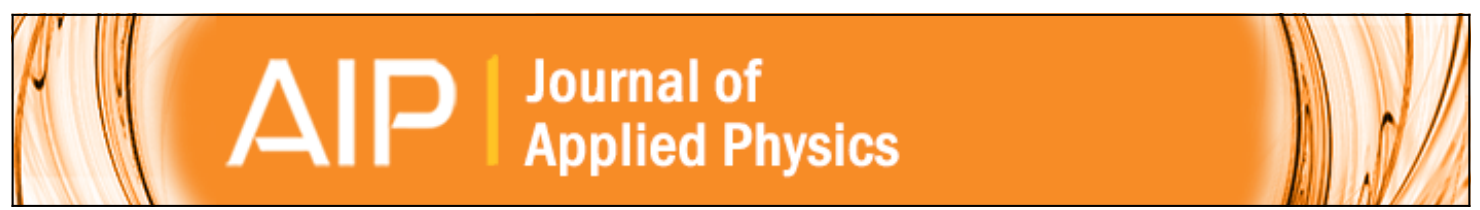

\section{Theory of Stress-Strain Relations in Anisotropic Viscoelasticity and Relaxation Phenomena}

M. A. Biot

Citation: Journal of Applied Physics 25, 1385 (1954); doi: 10.1063/1.1721573

View online: http://dx.doi.org/10.1063/1.1721573

View Table of Contents: http://scitation.aip.org/content/aip/journal/jap/25/11?ver=pdfcov

Published by the AIP Publishing

\section{Articles you may be interested in}

Nonlinear stress-strain relations for crystalline solids in initially deformed state

J. Appl. Phys. 112, 093501 (2012); 10.1063/1.4762000

Dynamic Stress-Strain Characteristics

J. Acoust. Soc. Am. 34, 715 (1962); 10.1121/1.1937156

Stress-Strain Relations in a Suspension of Dumbbells

Trans. Soc. Rheol. 1, 53 (1957); 10.1122/1.548808

Stress-Strain Rate Relations for Anisotropic Plastic Flow

J. Appl. Phys. 20, 15 (1949); 10.1063/1.1698231

The Stress-Strain Relation in Textile Fibers

J. Appl. Phys. 5, 53 (1934); 10.1063/1.1745230

\section{High Energy Nanosecond Lasers}

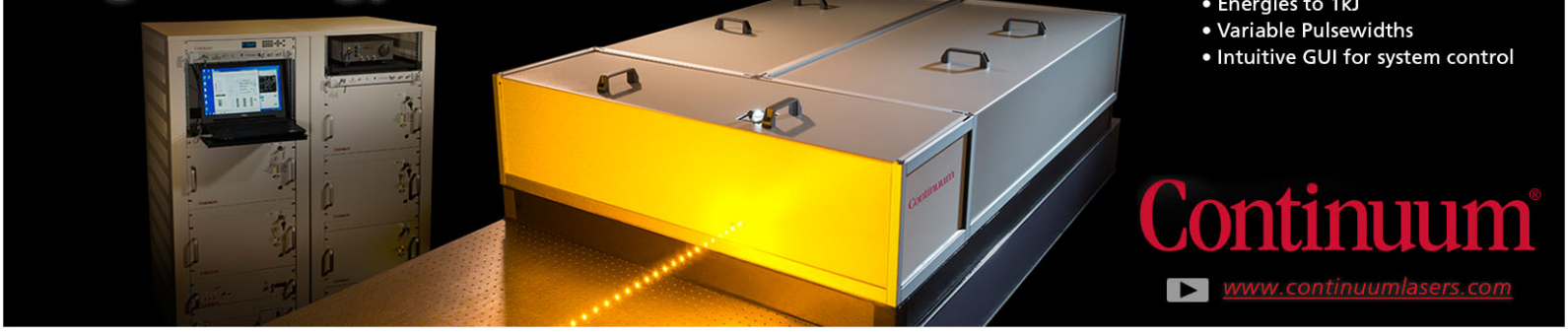




\title{
Theory of Stress-Strain Relations in Anisotropic Viscoelasticity and Relaxation Phenomena.
}

\author{
M. A. Brot* \\ Shell Development Company, New York, New York
}

(Received March 19, 1954)

\begin{abstract}
A thermodynamic derivation is given for the representation of a system having viscoelastic or relaxation properties by means of a potential and dissipation function familiar in Lagrangian mechanics. This leads to modes of relaxation which are used as normal coordinates to derive general expressions for operational tensors relating stress and strain. A large variety of phenomena involving interaction of diffusion, chemical reaction, heat transfer, mechanical deformation, etc., is included in this theory.
\end{abstract}

\section{INTRODUCTION}

$T$ $\mathrm{HE}$ behavior of systems having both elasticity and viscosity under applied forces or exhibiting relaxation is usually analyzed by considering a simple mechanical model constituted by interconnected springs and dashpots. An arbitrary system of this type made up of a lattice of points connected by such springs and dashpots may be considered as an $n$ degree of freedom system which is defined by two quadratic forms, the potential energy and the dissipation function. It is first shown in Sec. II by applying Onsager's theorem and the thermodynamics of irreversible phenomena that this model may represent the behavior of a very wide variety of phenomena in the process of their response to external action in the vicinity of equilibrium. Such phenomena may involve the application of external stresses, chemical reactions due to departure from chemical equilibrium, heat transfer, etc., and the coupling between these phenomena. The present treatment therefore includes Zener's theory of thermoelastic damping as a particular case. Also included are the effects of a liquid solvent which have been found experimentally in rock creep tests. We show that we may extend the concepts of potential energy and dissipation function to cover all these cases leading to their treatment by the methods of Lagrangian mechanics.

Section III derives the existence of modes of relaxation, i.e., solutions for which all variables are proportional to a decreasing real exponential of time. Properties of decay constants and other properties of these modes such as orthogonality are examined. Use of these modes is made in Sec. IV in order to solve for the forces or external actions in terms of a limited number of external or observed coordinates, and it is found that the most general behavior of external coordinates may be represented by a model made of a number of Maxwell type materials plus a spring and a dashpot all in parallel.

Section V deals with the stress-strain relations of a continuum and introduces the concept of operational tensor as a generalization for the elastic moduli of the theory of elasticity.

\footnotetext{
* Consultant.
}

Section VI deals with the particular form of these tensors for special cases of symmetry of the material. The possibility of multiple symmetry in the same material depending on the strain rate is pointed out.

The results are also applicable to electric network theory and give expression for the direct and crossimpedances of a $2 k$ terminal $R C$ network. Extension to an $L C$ network is also quite straightforward as it amounts to replacing $p$ by $p^{2}$.

\section{THERMODYNAMIC DERIVATION OF THE FUNDAMENTAL EQUATION OF RELAXATION PHENOMENA}

We consider a general thermodynamic system with $n$ degrees of freedom defined by $n$ state variables $q_{i}$. These degrees of freedom are of a quite general nature. They may include coordinates, local temperatures, piezoelectric charges, concentrations such as induced by chemical or solubility processes, etc. Correlatively we assume that the system is under the action of external forces in the generalized sense denoted by $Q_{i}$ such that $Q_{i} d q_{i}$ represents the energy furnished to the system. These forces may be externally applied stresses, electromotive forces, or may result from deviations of Gibbs and chemical potential from the equilibrium state. No external forces are associated with the temperature variables.

We also assume the system to be linear, i.e., that the coordinates $q_{i}$ and their derivatives are linear functions of the forces $Q_{i}$. The system includes a large reservoir at a constant temperature $T$ and the total system is closed, i.e., exchanges no heat with the outside. Under those circumstances the entropy and the internal energy are quadratic functions of the state variables.

We define the zero level of the entropy for the state where all variables are zero and $T$ is the uniform temperature of this equilibrium state. Part of the state variables will be local temperature increments concentration increments, etc. Where no forces are applied, the entropy of the system in the vicinity of the zero level is a maximum and we may write

$$
T S=-\frac{1}{2} \sum^{j} a_{i j} q_{i} q_{j}=-V,
$$

where $V$ is a positive definite quadratic form. The 
constant temperature $T$ is introduced here as a factor simply for convenience. When forces are applied this is replaced by

$$
T S=-\frac{1}{2} \sum^{i j} a_{i j} q_{i} q_{j}+\sum^{i} \beta_{i} q_{i} .
$$

If the system is displaced from the zero level by applying the forces very slowly so that the system is displaced reversibly by a succession of equilibrium states, expression (2.2) would constantly satisfy the conditions that the entropy is a maximum, namely,

$$
\partial S / \partial q_{i}=0 .
$$

We now investigate the significance of the linear terms in expression (2.2) for the entropy. We separate the system into two parts, a system I to which the forces $Q_{i}$ are applied and a system II constituted by the large reservoir at the constant temperature $T$. Conservation of energy requires that the total heat absorbed by system $I$ is

$$
d h=d U_{1}-\sum^{i} Q_{i} d q_{i}
$$

where $U_{\mathrm{I}}$ is the internal energy of $I$. Hence the reservoir acquires an increment of entropy

$$
d S_{\mathrm{II}}=-\frac{d U_{\mathrm{I}}}{T}+\sum^{i} \frac{Q_{i} d q_{i}}{T} .
$$

Denoting by $S_{\mathrm{I}}$ the entropy of system I we have for the total entropy

$$
d S=d S_{\mathrm{I}}-\frac{d U_{\mathrm{I}}}{T}+\sum^{i} \frac{Q_{i} d q_{i}}{T} .
$$

Now in the vicinity of the origin $q_{i}=0$, for a reversible process in which the forces $Q_{i}$ are constantly in equilibrium with the system, we have

$$
d S_{\mathrm{I}}=\frac{d U_{\mathrm{I}}}{T}-\sum^{i} Q_{i} d q_{i}
$$

But at the origin equilibrium requires $Q_{i}=0$ by defnition, hence at the origin

We conclude for $q_{i}=0$,

$$
d S_{\mathrm{I}}=\frac{d U_{\mathrm{I}}}{T} .
$$

$$
T \frac{\partial S}{\partial q_{i}}=Q_{i}=\beta_{i}
$$

Expression (2.2) for the total entropy becomes

$$
T S=-\frac{1}{2} \sum^{i j} a_{i j} q_{i} q_{j}+\sum^{i} Q_{i} q_{i} .
$$

By putting equal to zero the derivatives $\partial S / \partial q_{i}$ we obtain $n$ linear relations between the forces and the coordinates which correspond to equilibrium conditions of the system under those forces.

We now apply Onsager's theorem ${ }^{1}$ to expression (2.10). This theorem is concerned with the case where the derivatives $\partial S / \partial q_{i}$ are not zero, i.e., where we have a departure from equilibrium. These derivatives are considered as forces associated with time rates of change of the state variables $q_{i}$. The theorem states that these rates are linear functions of these derivatives and that the coefficients constitute a symmetric matrix. We write

$$
T \frac{\partial S}{\partial q_{i}}=\frac{1}{2} \sum^{j} b_{i j} \dot{q}_{i}
$$

with $b_{i j}=b_{j i}$. Since

$$
T \sum \frac{\partial S}{\partial q_{i}} \dot{q}_{i}=\frac{1}{2} \sum^{i j} b_{i j} \dot{q}_{i} \dot{q}_{j}=D
$$

is proportional to the rate of change of entropy which is always positive, we conclude that the quadratic form $D$ is positive definite.

The fundamental relation between forces and state variables may therefore be written in the general form,

$$
\sum^{j} a_{i j} q_{j}+\sum^{j} b_{i j} \dot{q}_{j}=Q_{i} .
$$

These equations may also be written in the familiar Lagrangian form

$$
\frac{\partial V}{\partial q_{i}}+\frac{\partial D}{\partial \dot{q}_{i}}=Q_{i}
$$

We see that $V$ plays the role of a potential energy and $D$ that of a dissipation function. The system therefore is represented by a mechanical model of springs and dashpots connecting two by two a lattice of $n / 3$ spacially distributed points with $n$ independent coordinates and corresponding forces $Q_{i}$ applied at these points. For simplicity we shall refer hereafter to the state variables $q_{i}$ as coordinates.

\section{RELAXATION MODES AND RELAXATION SPECTRUM}

The general equations (2.13) are written for convenience

with

$$
\begin{gathered}
\sum^{j} A_{i j} q_{j}=Q_{i} \\
A_{i j}=a_{i j}+p b_{i j}
\end{gathered}
$$

and the time operator

$$
p=d / d t \text {. }
$$

For studying the properties of these equations it is

${ }^{1}$ S. R. De Groot, Thermodynamics of Irreversible Processes (Interscience Publishers Inc., New York, 1952). 
important to treat first the homogeneous equations

$$
\sum^{j} A_{i j} q_{j}=0 \text {. }
$$

If $p$ is considered as a parameter we see that the homogeneous equations lead to an eigenvalue problem. An algebraic equating for the characteristic values of $p$ is obtained by equating to zero the determinant of the coefficients $A_{i j}$,

$$
\Delta=\operatorname{det}\left(A_{i j}\right)=0 .
$$

For the sake of generality we shall say that this equation is of the $n$th degree and that there are $n$ roots but will consider that in addition to possible multiple and zero roots some of the roots may be infinite. We denote the roots of equation (3.5) by $-\lambda_{8}$. Let us assume first that the roots are all distinct, finite, and different from zero. The general solution of the homogeneous Eqs. (3.4) is then

$$
q_{j}=\sum^{s} \phi_{j}^{(s)} e^{-\lambda_{s} t}
$$

To each root $-\lambda_{\varepsilon}$ corresponds a solution

$$
q_{j}^{(s)}=\phi_{j}^{(s)} e^{-\lambda_{z} t}
$$

which is called a relaxation mode. That the roots $-\lambda_{s}$ are real and negative is easily seen as follows. We consider two modes

$$
\begin{aligned}
& q_{j}^{(s)}=\phi_{j}^{(s)} e^{-\lambda_{t} t}, \\
& q_{j}^{(r)}=\phi_{j}^{(r)} e^{-\lambda_{r} t}
\end{aligned}
$$

corresponding to the two roots $-\lambda_{s},-\lambda_{r}$. That these solutions are orthogonal is established by the usual procedure of substituting each solution in the equation then multiplying each equation for the solutions $\phi_{j}^{(s)}$ by $\phi_{i}{ }^{(r)}$ and those for $\phi_{j}{ }^{(r)}$ by $\phi_{i}{ }^{\left({ }^{(8)}\right.}$. Adding each set of equations gives the two relations

$$
\begin{gathered}
\sum^{i j} a_{i j} \phi_{j}{ }^{(s)} \phi_{i}{ }^{(r)}-\lambda_{s} \sum^{i j} b_{i j} \phi_{j}{ }^{(s)} \phi_{i}{ }^{(r)}=0, \\
\sum^{i j} a_{i j} \phi_{j}{ }^{(r)} \phi_{i}{ }^{(s)}-\lambda_{r} \sum^{i j} b_{i j} \phi_{j}{ }^{(r)} \phi_{i}^{(s)}=0 .
\end{gathered}
$$

Because of the symmetry $a_{i j}=a_{j i}$ and $b_{i j}=b_{j i}$ the quadratic forms are the same in both equations, and by subtraction of Eq. (3.9) we find that if $\lambda_{s}$ and $\lambda_{r}$ are distinct, this implies

$$
\sum^{i j} a_{i j} \phi_{j}{ }^{(s)} \phi_{i}{ }^{(r)}=\sum^{i j} b_{i j} \phi_{j}{ }^{(s)} \phi_{i}{ }^{(r)}=0 .
$$

These equations express the orthogonality of the modes. It is easily verified that they cannot be satisfied if the modes are complex conjugates because of the property that the quadratic forms are positive. So the modes are real. Moreover if we put $s=r$ in (3.9) we find

$$
\lambda_{s}=\frac{\sum^{i j} a_{i j} \phi_{i}{ }^{(s)} \phi_{j}{ }^{(s)}}{\sum^{i j} b_{i j} \phi_{i}{ }^{(s)} \phi_{j}{ }^{(s)}}=\frac{V}{D},
$$

where $V$ and $D$ are positive definite forms corresponding to the potential energy $V$ and the dissipation function $D$ (in which we replace $q_{i}$ by $\left.\phi_{i}^{\left({ }^{(s)} t\right.}\right)$. Therefore, all roots $-\lambda_{s}$ are real and negative.

Now these properties are not restricted to the case where all roots are distinct, finite, and different from zero. In case of a multiple root $-\lambda_{s}$ of multiplicity $\alpha$, we may determine $\alpha$ orthogonal modes corresponding to this root. Also, in the case of an infinite root we have a mode which is simply a column of constants independent of time. The case of a zero root corresponds to a solution of the type

$$
q^{(8)}=\phi_{j}^{\left({ }^{(s)}\right.} t .
$$

This will be further clarified by considering the system from the standpoint of normal coordinates in the next section.

In most physical systems a great many variables are "hidden," i.e., we do not observe them. External forces are only applied to $k$ coordinates $q_{1} \cdots q_{k}$ while the $n-k$ others constitute what we shall call the internal system. This is expressed by writing Eqs. (3.1) in the form

$$
\left[\begin{array}{c:c}
N & S \\
\hdashline T & M \\
\hdashline
\end{array}\right]\left[\begin{array}{c}
q_{1} \\
q_{k} \\
q_{k+1} \\
\cdot \\
q_{n}
\end{array}\right]=\left[\begin{array}{c}
Q_{1} \\
Q_{k} \\
0 \\
\cdot \\
0
\end{array}\right] .
$$

The matrix $M$ represents an internal subsystem of $n-k$ degrees of freedom which is of the same general nature as the total system with its own $n-k$ relaxation constants $\sigma_{8}$ and modes corresponding to roots $-\sigma_{s}$ of the determinant of the submatrix $[M]$,

$$
M=\operatorname{det}[M]=0 \text {. }
$$

The relaxation constants $\lambda_{s}$ correspond to a set of relaxation times which if a great many degrees of freedom are considered may be said to constitute a spectrum. The spectrum $\sigma_{\varepsilon}$ of a subsystem will depend of course on the particular subsystem which is considered as internal. It is also a consequence of the properties of the type of matrices involved that the roots $\sigma_{s}$ are located between the roots $\lambda_{s}$ because the system defined by the matrix $M$ may be considered as the original system of determinant $\Delta$ in which $k$ restraints, $q_{1}=q_{2}=\cdots q_{k}=0$, have been introduced.

\section{NORMAL COORDINATES AND GENERALIZED FORCES}

We perform a change of coordinates from $q_{i}$ to $\xi$, by the $n$ linear relations

$$
q_{i}=\sum^{s} \phi_{i}^{(s)} \xi_{s},
$$

where $\phi_{i}{ }^{(s)}$ is the modal column of the sth relaxation mode. The $\xi$ 's are called the normal coordinates. The 
Lagrangian Eq. (2.14) then becomes

$$
\frac{\partial V}{\partial \xi_{s}}+\frac{\partial D}{\partial \xi_{s}}=\Xi_{s}
$$

where the generalized normal force $\Xi$, corresponding to the coordinate $\xi_{\mathrm{s}}$ is given by

$$
\Xi_{s}=\sum^{j} \phi_{j}^{(s)} Q_{j}
$$

We normalize the $\phi_{j}^{(s)}$ columns in such a way that for each $s$

$$
\sum^{i j} b_{i j} \phi_{i}^{(s)} \phi_{j}{ }^{(s)}=1
$$

except for the case where $\lambda_{s}=\infty$ where we normalize by

$$
\sum^{i j} a_{i j} \phi_{i}^{(s)} \phi_{j}^{(s)}=1 \text {. }
$$

Then we may write for $\lambda_{8} \neq \infty$,

and for $\lambda_{0}=\infty$,

$$
\begin{aligned}
& V=\frac{1}{2} \sum^{8} \lambda_{8} \xi_{s}{ }^{2}, \\
& D=\frac{1}{2} \sum^{8} \xi_{s}{ }^{2}
\end{aligned}
$$

$$
\begin{aligned}
& V=\frac{1}{2} \sum^{6} \xi_{8}{ }^{2}, \\
& D=0 .
\end{aligned}
$$

The Lagrangian equations for the normal coordinates are thus the $n$ equations

$$
\begin{aligned}
\left(p+\lambda_{8}\right) \xi_{8} & =\Xi_{8}, \\
\xi_{8} & =\Xi_{8},
\end{aligned}
$$

where the last equation corresponds to modes of infinite root $\lambda_{s}$. These are $n$ equations which are quite general and may correspond to any case of multiplicity of $\lambda_{8}$ or to zero and infinite roots. In the case of $\alpha$ multiplicity of a root $\lambda_{s}$ the corresponding Eq. (4.8) is simply repeated $\alpha$ times.

Equations (4.8) are immediately solved in terms of the coordinates

$$
\begin{aligned}
& \xi_{8}=\frac{\Xi_{8}}{p+\lambda_{8}}, \\
& \xi_{s}=\Xi_{s} .
\end{aligned}
$$

With these results we may solve the fundamental Eq. (3.1) for the variables $q_{j}$ in terms of the forces $Q_{j}$. Substituting $\xi_{\propto}$ in (4.1) and using (4.3) for $\Xi_{8}$, we find

$$
q_{i}=\sum_{j=1}^{k} Q_{j}\left[\sum \frac{C_{i j}^{(s)}}{p+\lambda_{s}}+C_{i j}\right] .
$$

The summation $\dot{\Sigma}$ is extended to all the distinct roots $\lambda_{\text {s. }}$

$$
C_{i j}^{(s)}=\phi_{i}{ }^{(s)} \phi_{j}^{(s)}
$$

if $\lambda_{s}$ is a single root and

$$
C_{i j}{ }^{(s)}=\dot{\sum} \phi_{i}{ }^{(s)} \phi_{j}{ }^{(s)}
$$

is the summation extended to all the modes of the multiplicity $\alpha$ if $\lambda_{s}$ is a multiple root. The value of $C_{i j}$ is the same expression as (4.11) and (4.12) corresponding to the infinite roots, single or multiple. From these expressions it is seen that the diagonal terms $C_{i i}{ }^{(s)}$ and $C_{i i}$ are always positive and that the coefficients are symmetric, i.e.,

$$
C_{i j}{ }^{(s)}=C_{j i}^{(s)}, \quad C_{i j}=C_{j i} .
$$

In expression (4.10) the summation is extended from $j=1$ to $j=k$ over all forces $Q_{j}$ which are taken different from zero. The $k$ first equations therefore give the observed variables $q_{1}$ and $q_{k}$ in terms of the forces $Q_{1}$ to $Q_{k}$ applied to those coordinates.

Now we would like to solve these equations back and express the $k$ applied forces in terms of the observed coordinates. This is best done by going back to the original Eqs. (3.13).

We consider the subsystem represented by the equation

$$
[M]\left[\begin{array}{c}
q_{k+1} \\
q_{n}
\end{array}\right]=0 .
$$

This system may be represented by its $n-k$ normal coordinates $\xi_{k+1} \cdots \xi_{n}$. The complete $n$ degree of freedom system may be represented by these normal coordinates and by the $k$ coordinates $q_{1} \cdots q_{k}$. The potential energy and the dissipation function for this system may then be written

$$
\begin{aligned}
& V=\frac{1}{2} \sum^{i j} a_{i j} q_{i} q_{j}+\sum^{i s} a_{i s}{ }^{\prime} q_{i} \xi_{s}+\frac{1}{2} \dot{\sum} \sigma_{r} \xi_{s}{ }^{2} \\
& D=\frac{1}{2} \sum^{i j} b_{i j} \dot{q}_{i} \dot{q}_{j}+\sum^{i s} b_{i s}{ }^{\prime} \dot{q}_{i} \xi_{s}+\frac{1}{2} \sum^{s} \dot{\xi}_{\mathrm{s}}{ }^{2}
\end{aligned}
$$

where $i$ and $j$ vary from 1 to $k$ and $s$ from $k+1$ to $n$. As before we note that if one of the roots $\sigma_{s}$ of $M$ is infinite, the normalization of the mode can be done in such a way that the corresponding $\xi_{s}^{2}$ term in the dissipation function does not appear while there is a term $\xi_{s}{ }^{2}$ in the potential energy. In that case, however, the condition that $D$ is positive definite also requires that all coefficient $b_{i i^{\prime}}$ corresponding to that mode vanish. Similarly since $V$ is also positive definite if there are zero values of $\sigma_{s}$ all corresponding coefficients $a_{i s}{ }^{\prime}$ must be zero.

With normal coordinates for the subsystem, the 
equations for the complete system become

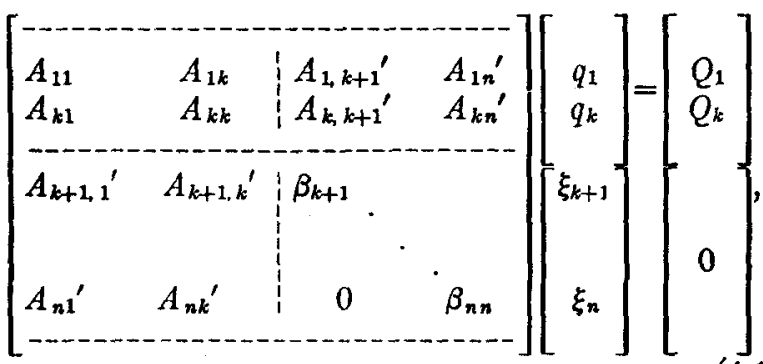

where $A_{i j}=a_{i j}+p b_{i j}, A_{i s}{ }^{\prime}=a_{i s}{ }^{\prime}+p b_{i s}{ }^{\prime}$ and $\beta_{s}=p+\sigma_{s}$ or 1 , depending on whether $\sigma_{s}$ is finite or infinite. We now solve the last $n-k$ equations for $\xi_{8}$. If $\sigma \neq \ldots, \infty$, we may write

$$
\xi_{a}=-\sum_{j=1}^{k} \frac{A_{s j} q_{j}}{p+\sigma_{s}}
$$

and, if $\sigma_{s}=\infty$,

$$
\xi_{s}=-\sum_{j=1}^{k} A_{s i}^{\prime} q_{j}=-\sum_{j=1}^{k} a_{s j}^{\prime} q_{j} .
$$

We also note that, if $\sigma_{s}=0$,

$$
\xi_{a}=-\sum_{j=1}^{k} b_{s j}^{\prime} q_{j}
$$

Substituting these values of $\xi$ in the first $k$ equations, we find

with

$$
Q_{i}=\sum^{j} T_{i j} q_{j}
$$

$$
T_{i j}=A_{i j}-\sum \frac{A_{i s}{ }^{\prime} A_{s j}{ }^{\prime}}{p+\sigma_{s}} .
$$

If $\sigma$, is zero or infinite for any particular mode the last term is replaced, respectively, by

$$
\begin{array}{r}
A_{i s}{ }^{\prime} A_{s j}{ }^{\prime}=A_{i s}{ }^{\prime} b_{s j}{ }^{\prime}, \\
A_{i s}{ }^{\prime} A_{s j^{\prime}} / p+\sigma_{s}=A_{i s}{ }^{\prime} a_{s j}{ }^{\prime} .
\end{array}
$$

All coefficients in (4.20) are symmetric

$$
T_{i j}=T_{j i} \text {. }
$$

The coefficients may be written

where

$$
T_{i j}=\dot{\sum} \frac{p}{p+\sigma_{z}} D_{i j}{ }^{(*)}+D_{i j}+D_{i j}{ }^{\prime} p,
$$

$$
\begin{aligned}
& D_{i j}^{(s)}=\psi_{i}^{(s)} \psi_{j}^{(s)} \text {, } \\
& D_{i j}=a_{i j}-\sum \frac{a_{i s}{ }^{\prime} a_{s j}{ }^{\prime}}{\sigma_{s}}, \\
& D_{i j}{ }^{\prime}=b_{i j}-\sum^{s} b_{i s}{ }^{\prime} b_{s j}{ }^{\prime}, \\
& \psi_{i}^{(s)}=a_{i s}^{\prime} / \sigma_{i}^{t}-\sigma_{i}{ }^{t} b_{i a^{\prime}} \text {. }
\end{aligned}
$$

All diagonal terms of the matrix $T_{i j}$ are positive. This may be seen directly from the expression (4.26) for $D_{i i}{ }^{(g)}$. As regards $D_{i i}$ and $D_{i i}{ }^{\prime}$ they must be positive because the ratio $Q_{i} / q_{i}$ and $Q_{i} / \dot{q}_{i}$ must be positive for $p=0$ and $p=\infty$, respectively. Expression (4.25) of $T_{i j}$ shows that any relaxation phenomena may be represented by a spring, a dashpot, and a sum of a great many elements made up of a Maxwell type material corresponding to the operator

$$
\left(p / p+\sigma_{s}\right) D_{i j}^{(s)} \text {. }
$$

Such a Maxwell material is represented by a spring and dashpot in series. We thus have shown that a mechanical model of springs and dashpots in series and in parallel may represent a very wide variety of relaxation effect involving not only mechanical, but chemical, thermodynamic phenomena, etc., and coupled effects between them.

\section{APPLICATION TO THE GENERAL STRESS-STRAIN RELATIONS IN AN ANISOTROPIC CONTINUUM}

We shall apply the previous theory to the formulation of the stress-strain relations in a viscoelastic material or more generally to any material exhibiting relaxation effects. We assume the deformation of this material under stress to be made of the variation of many degrees of freedom both internal and external. The internal degrees of freedom may be of mechanical, chemical, or other physical nature. In this case the nine stress components

$$
\sigma_{\mu \nu}=\left\{\begin{array}{lll}
\sigma_{x x} & \sigma_{x y} & \sigma_{x z} \\
\sigma_{y x} & \sigma_{y y} & \sigma_{y z} \\
\sigma_{z x} & \sigma_{x y} & \sigma_{z z}
\end{array}\right\}
$$

applied to the faces of an elementary cube of material play the role of applied generalized forces $Q_{1} \cdots Q_{k}$ $(k=9)$ considered in the previous section. The nine corresponding observed coordinates $q_{1} \cdots q_{k}$ are the nine components of the strain tensor,

$$
e_{\mu \nu}=\left\{\begin{array}{lll}
e_{x x} & e_{x y} & e_{x z} \\
e_{y x} & e_{y y} & e_{y z} \\
e_{z x} & e_{z y} & e_{z z}
\end{array}\right\} .
$$

Because of the symmetry

$$
\sigma_{\mu \nu}=\sigma_{\nu \mu,} \quad e_{\mu \nu}=e_{\nu \mu}
$$

we have only six independent components of these tensors. We now apply relations (4.20) and (4.25) to this material and write

with

$$
\begin{gathered}
\sigma_{\mu \nu}=\sum^{i j} P_{\mu \nu}{ }^{i i} e_{i j} \\
P_{\mu \nu}{ }^{i j}=\sum \frac{p D_{\mu \nu}{ }^{i j(s)}}{p+\sigma_{s}}+D_{\mu \nu}{ }^{i j}+p D_{\mu \nu}{ }^{i j} .
\end{gathered}
$$

This is an operational tensor having the following sym- 
metry properties :

$$
P_{\mu \nu}^{i j}=P_{\nu \mu}^{i j}=P_{\mu \nu}^{j i}, \quad P_{\mu \nu}^{i j}=P_{i j}^{\mu \nu} .
$$

These properties are the same as those of the elastic moduli of the theory of elasticity and we may therefore treat these operators in a similar way. By solving these equations with respect to the strain, we shall obtain a similar expression with an operator having the same form as in Eq. (4.10).

In expression (5.5) the summation is extended to all internal relaxation constants $\sigma_{s}$. Since the solid has a great many internal degrees of freedom there may be in some cases an almost continuous distribution of relaxation modes. This will correspond to a spectrum or spectral density distribution of the relaxation constant. With a density distribution function $\gamma(\sigma)$ we may write

$$
P_{\mu \nu}{ }^{i j}=p \int_{0}^{\infty} \frac{D_{\mu \nu}{ }^{i j}(\sigma) \gamma(\sigma)}{p+\sigma} d \sigma+D_{\mu \nu}{ }^{i j}+p D_{\mu \nu}{ }^{i i}
$$

This operational tensor is an analytic function of the time operator $p$. The more general representation of the operational tensors $P_{\mu \nu}{ }^{i i}$ is therefore a set of 21 analytic functions of $p$ constituting a symmetric six by six matrix. Under the integral, due account must be taken of the possibility of degeneracy of the modes in evaluating the product $D_{\mu \nu}{ }^{i j}(\sigma) \gamma(\sigma)$. From expressions (4.26) we see that we may write

$$
D_{\mu \nu}^{i j}(\sigma)=\psi_{\mu \nu}(\sigma) \psi^{i j}(\sigma) .
$$

\section{SPECIAL CASES OF SYMMETRY}

The operational equations (5.4) may be simplified if we introduce the assumption that the material has a certain degree of symmetry. We shall discuss two examples, the case of cubic symmetry and the case of isotropy.

We proceed as in the classical theory of elasticity, where in the present case the operational tensor plays the same role as the elastic moduli. The relation between stress and strain must remain invariant under certain symmetry operations. In elasticity a cubic crystal has three elastic constants. Similarly in our case cubic symmetry leads to three operational tensor components. If we take the coordinate axes along the cubic axes the stress strain relations become

$$
\begin{aligned}
& \sigma_{x x}=2 Q e_{x x}+R e, \\
& \sigma_{y y}=2 Q e_{y y}+R e, \\
& \sigma_{z z}=2 Q e_{x z}+R e, \\
& \sigma_{x y}=2 S e_{x y}, \\
& \sigma_{y z}=2 S e_{y z}, \\
& \sigma_{z x}=2 S e_{z x},
\end{aligned}
$$

with $e=e_{x x}+e_{y y}+e_{z z}$. The three operators are

$$
\begin{aligned}
& Q=\dot{\sum} \frac{p Q^{s}}{p+\sigma_{s}}+Q+Q^{\prime} p, \\
& R=\sum \frac{p R^{s}}{p+\sigma_{s}}+R+R^{\prime} p, \\
& S=\sum \frac{p S^{s}}{p+\sigma_{s}}+S+S^{\prime} p .
\end{aligned}
$$

The quantities $Q^{g}, Q, Q^{\prime}, R^{s}, \cdots$ etc. are characteristic constants of the material, and $\sigma_{s}$ are the internal relaxation constants. The summation may be replaced by an integration with a spectral distribution.

In the case of isotropy the number of operators is further restricted. The condition of invariance under any rotation implies

$$
Q=S
$$

and the stress-strain relations for the isotropic case become

$$
\begin{aligned}
& \sigma_{x x}=2 Q e_{x x}+R e, \\
& \sigma_{y y}=2 Q e_{y y}+R e, \\
& \sigma_{z z}=2 Q e_{z z}+R e, \\
& \sigma_{x y}=2 Q e_{x y}, \\
& \sigma_{y z}=2 Q e_{y z}, \\
& \sigma_{z x}=2 Q e_{z x} .
\end{aligned}
$$

The two operators $Q$ and $R$ are invariants of the form (6.2) and are the formal analogs of the Lame constants in the theory of elasticity. The case of hydrostatic stress is obtained by putting

with

$$
\begin{aligned}
& \sigma_{x x}=\sigma_{y y}=\sigma_{z z}, \\
& \sigma_{x y}=\sigma_{y z}=\sigma_{z x}=0,
\end{aligned}
$$

we find

$$
3 \sigma=\sigma_{x x}+\sigma_{y y}+\sigma_{z g},
$$

$$
\sigma=\left(\frac{2}{3} Q+R\right) e .
$$

The operator $\frac{2}{3} Q+R$ is equivalent to a bulk modulus. The existence of this operator in the general form correspond to the assumption of bulk relaxation or volume creep. This may of course occur at high pressure or for porous materials. If $\frac{2}{3} Q+R$ is a constant, then the material obeys Hooke's law under hydrostatic pressure. Denoting by $K$ the elastic bulk modulus this requires a relation between the operators $Q$ and $R$,

$$
\frac{2}{3} Q+R=K \text {. }
$$

Equations (6.4) are then expressed by means of a single operator $Q$,

$$
\begin{aligned}
& \sigma_{x x}=2 Q e_{x x}+\left(K-\frac{2}{3} Q\right) e, \\
& \sigma_{x y}=2 Q e_{x y} .
\end{aligned}
$$

Very often the assumption is made that the material is 
incompressible. In this case the operator $R$ is infinite and $e=0$, but the product $R e$ is finite and the limiting relation $(6.7)$ is

$$
\sigma=\operatorname{Re} .
$$

It is convenient to introduce this negative hydrostatic pressure $\sigma$ in the relations (6.4) and write

$$
\begin{aligned}
\sigma_{x x}-\sigma & =2 Q e_{x x}, \\
\sigma_{y y}-\sigma & =2 Q e_{y y}, \\
\sigma_{z z}-\sigma & =2 Q e_{z z}, \\
\sigma_{x y} & =2 Q e_{x y}, \\
\sigma_{y z} & =2 Q e_{y z}, \\
\sigma_{z y} & =2 Q e_{z x} .
\end{aligned}
$$

In this case also a single operator $Q$ is necessary to characterize the material.

There is need to call attention to a difference between the operational stress-strain relations and the theory of elasticity with respect to symmetry properties. This is the possibility of occurrence of multiple or mixed symmetry. If we note that $P_{\mu \nu}{ }^{i i}$ is written in terms of the tensor

$$
D_{\mu \nu}^{i j(s)} \quad D_{\mu \nu}^{i j} \quad D_{\mu \nu}^{\prime i j},
$$

it is not contradictory to assume that each of these tensors has its own symmetry. The material would then exhibit different symmetries, each of which would predominate at various strain rates.

\section{ACKNOWLEDGMENTS}

The author is indebted to the Shell Development Company for permission to publish the present work which has been sponsored during the past years as part of a research program on the mechanical properties of rock at the Exploration and Production Research Division in Houston, Texas.

\title{
Electrical Noise Pulses from Polarized Dielectrics*
}

\author{
N. P. Baumann and G. G. Wiseman \\ University of Kansas, Larerence, Kansas
}

(Received April 5, 1954)

\begin{abstract}
Electrical noise pulses have been observed at elevated temperatures in polarized dielectrics (electrets) made of carnauba wax and of a vinyl acetate polymer. Pulse rate versus time was observed for electrets heated at a uniform rate and the results differ from those previously reported by others. Experiments devised to determine the origin of the pulses indicate that they are associated with the decay of volume polarization rather than with real surface charge. The total charge associated with the pulses from carnauba wax electrets is at least 5 percent of the maximum electret charge.
\end{abstract}

\section{INTRODUCTION}

$\mathrm{E}$ LECTRICAL noise pulses from electrets were first reported by Gutmann ${ }^{1}$ but his mode of detection and the nature of his results were not given. Boyer ${ }^{2}$ measured random electrical noise pulses in a number of plastics which were subjected to fields of $4 \mathrm{kv} / \mathrm{cm}$ to $118 \mathrm{kv} / \mathrm{cm}$. More recently, Kojima and Kato investigated noise pulses from electrets which were being heated at a uniform rate from room temperature to a temperature high enough to destroy the electret's charge. Kojima and Kato used two electrets which had been prepared by subjecting identical specimens of a carnauba wax and rosin mixture to the same electrical and thermal treatment. One electret was used for observing the noise pulses, the other for measuring the surface charge.

\footnotetext{
* Supported by the U. S. Signal Corps.

1 F. Gutmann, Revs. Modern Phys. 20, 457 (1948). This article reviews experimental and theoretical work on electrets. It contains an extensive bibliography.

${ }_{2}^{2}$ R. F. Boyer, J. Appl. Phys. 21, 469 (1950).

${ }^{3}$ S. Kojima and K. Kato, J. Phys. Soc. (Japan) 6, 207 (1951).
}

The present work was undertaken to verify and extend the work of Kojima and Kato. In this study, however, both the noise pulses and the charge measurements were made on the same electret primarily because incidental experiments revealed that electrets which have been given apparently identical thermal and electrical treatment are often unlike. Moreover, surface charge measurements were made by the conventional dissectible capacitor method, ${ }^{4,5}$ rather than by the induction voltmeter method used by Kojima and Kato because the dissectible capacitor method allows a much smaller air gap between the measuring electrode and the electret. The average electric field $E$ causing depolarization within a "short-circuited" electret with one air gap is (in mks units)

$$
E=\sigma / \epsilon_{0}\left(d_{2} / d_{1}\right)+K
$$

where $\sigma$ is the net surface charge of the electret, $d_{1}$ is

\footnotetext{
${ }^{4}$ M. Eguchi, Phil. Mag. 49, 178 (1925)

5 B. Gross, J. Chem. Phys. 17, 866 (1949). This paper presents a two-charge theory of electrets.
} 